\title{
COMPARING THE EXPERIENCES OF WOMEN IN UNDERGRADUATE ENGINEERING ACROSS DIFFERENT SCHOOLS
}

\author{
Natalie Mazur, Bronwyn Chorlton, and John Gales* \\ York University \\ *jgales@yorku.ca
}

\begin{abstract}
A previous study by the authors that was conducted on students in accredited undergraduate engineering programs showed significant differences between men's and women's experiences in their programs. That previous first-stage study highlighted that the causes of Canada's low retention of women in the field may be at least partially attributable to women's negative experiences at the beginning of their careers, in their undergraduate education. The research done thus far by the authors was largely explorative; there is now a need to begin identifying where and when students are experiencing negativity tied to their gender. The purpose of the research herein is to get a more comprehensive understanding of how specific behaviours and practices of professors, teaching assistants, peers, and other personnel in the classroom affect students will have significant consequences for what inclusive pedagogy in engineering should look like. As a part of this, the previous pilot study was revised, expanded and distributed to four accredited engineering institutions in North America. The results of the present study reinforce authors' previous theories and indicate that across the institutions surveyed, peers and professors made up the primary source of discouragement and intimidation against students. In addition, institutional differences uncovered in this study suggest that specific programs and initiatives at the institutions in question at least partially affect student experiences, and later their retention in the field. The authors conclude that institutions need to begin targeting peers and professors for equity education, bias eradication training, and other initiatives.
\end{abstract}

Keywords: diversity, STEM, WSTEM, women, gender, undergraduate, education, inclusivity

\section{INTRODUCTION}

The percentage of students enrolling in undergraduate engineering programs in Canada who are women has been very slowly increasing over the past 10 years [9]. However, this statistic does not hold for all engineering disciplines, with some disciplines seeing decreases in women's representation in those fields [9]. Almost 22\% of engineering undergraduates are women - a proportion that drops to $17 \%$ for newly licensed engineers [10]. This issue of retention continues throughout women's career trajectories in engineering. This necessitates greater research focus, to understand the factors that may be influencing women to leave the engineering profession. Although there have been many efforts on the recruitment end, if women are not staying in the field, then these efforts will have been ineffective. Tackling recruitment alone is not enough to encourage increases in diversity $[2,7]$.

Numerous studies have shown that women face more obstacles than men throughout their careers; this retention issue has often been termed the "Leaky Pipeline" [5, 6, 14]. A key variable that has been responsible for perpetuating these obstacles is what has often been called a "Chilly Climate" - a hypermasculine, male-dominated, and sexist environment [1, 5, 8, 11, 12]. Women have consistently reported being intimidated, being targets of subtle sexist humour, and being regarded as 'tokens' or 'diversity hires' who have been hired to fill a quota as opposed to because of their abilities. The chilly climate is not something unique to the workplace. It is introduced and perpetuated by undergraduate institutions [17], leaving women in undergraduate programs disillusioned and excluded $[5,13$, 15]. Most of this highlighted research has been done in the United States, but little to none exists on the Canadian cohort. The Canadian schooling systems and licensing laws differ from those of the United States, so it is necessary to begin understanding the local situation. There may be cultural variables responsible for retention issues in Canada that are unique. Without accounting for those, we run the risk of relying on unrepresentative data and recommendations that are irrelevant or ineffective for our situation.

A previous study by the authors on the experiences of different genders in engineering programs in Canada indicated significant differences in men's and women's experiences throughout their engineering programs [16]. That first-stage study highlighted that the causes of Canada's low retention of women in the field are at least partially attributable to women's negative experiences at the beginning of their careers, in their undergraduate 
education. The research done thus far has been explorative; there is now a need to begin identifying where and when students are experiencing negativity, and if it is correlated to their gender. Furthermore, previous results were based on a single school; different schools have different policies and climates that could contribute to student experiences in different ways [4]. Especially considering the fact that education decisions are made provincially as opposed to nationally in Canada, it is important to understand student experiences from many different contexts before making any sweeping generalizations and recommendations.

Understanding how specific behaviours and practices of professors, teaching assistants, peers, and other personnel in the classroom affect students will have significant consequences for what inclusive pedagogy in engineering should look like. A significant retention drop, or "leak in the pipeline," occurs between women's graduation from university and the years in which they prepare for licensure. If women have more negative experiences in their undergraduate programs than men, they have already overcome challenges that may further make resilience and retention difficult. Furthermore, if students at particular schools have more negative experiences than students at other schools, some graduates may enter their careers at a great disadvantage. Lessening negative experiences for all students, and allowing women to have an equal experience, is of utmost importance to ensure that engineers begin their careers strong and motivated, and that all have engineers have equal opportunities to move forward.

\section{METHODS}

The purpose of this research is to identify factors in engineering students' undergraduate education that may be contributing to their retention beyond the undergraduate degree. The primary target of this research is to identify challenges that affect genders and institutions in different ways. A 17-question survey was distributed to undergraduate engineering students at four accredited universities - three in Canada and one in the United States. All universities surveyed are public. The survey was made up of both multiple choice and open-ended questions. These questions were selected based on issues identified in previous research.

\subsection{Participants}

A total of 282 students completed the study - 68 from Institution A, 117 from Institution B, 36 from Institution C, and 61 from Institution D. Institution $B$ is an American public university, while Institutions $\mathrm{A}, \mathrm{C}$, and $\mathrm{D}$ are Canadian. The Canadian institutions were not the same institution as in the previous pilot study [16]. Participants were recruited via email and social media posts directed by administrative contacts at each institution. About $7 \%$ of all invitees participated. Of the 282 respondents, $55.9 \%$ identified as women and $40.47 \%$ identified as men. The remaining $3.63 \%$ identified as another gender or preferred not to indicate their gender. The respondents represented more than 18 streams of engineering and all four years of study (1-4). The average age of participants was 21.8 years old. All participants were invited to complete the survey over three to four weeks in December 2018. The email and social media posts explicitly stated the purpose of the survey and students of all genders and backgrounds were invited to participate. The survey obtained ethics clearance from the researchers' home university as well as the institutions being surveyed. The authors have chosen not to disclose the universities involved in publication.

\subsection{Materials}

The survey was made available online through Qualtrics Experience Management, a data collection platform. Each institution had a slightly different survey, with appropriate changes made to the name of the institution and the programs offered. Participants used a personal computer or other device connected to the internet to answer the questions. The survey was intended to be completed independently. The survey itself consisted of an informed consent form, given at the beginning of the survey, and 17 questions divided into four blocks according to theme (e.g., demographics, experiences). Questions were presented one block at a time (see Appendix A). All participants received the same questions in the same order. Where there were multiple possible answers in multiple choice questions, the order in which the answers were presented was randomized for each participant.

\subsection{Procedure}

Once participants navigated to the survey link on their device of choice, they read through the informed consent form. If they accepted the terms of their participation, they clicked the navigation button to give their consent to begin the survey. If they declined participation, they were free to close the browser.

The first block of questions in the survey related to demographic items such as engineering program, age, gender, and year standing. The next block asked questions related to student experiences. The final block consisted of an optional comments box if participants wanted to share any further information related to the survey. After completing the survey, participants' answers were submitted to the Qualtrics database.

\section{RESULTS}

Survey answers differed between genders and institutions. Chi square tests were used to determine whether significant relationships existed between 
responses to demographic questions and responses to educational experience questions. Results with $\mathrm{p}$ values less than 0.05 (i.e., a confidence level greater than 95\%) were considered to be statistically significant, as per common research standards [3].

In general, women showed similar trajectories of discouragement between all schools. Discouragement increased in middle years and dropped in fourth year. Figure 1 shows that the fourth year drop is slightly more prominent for women than for men, and that on average a significantly higher proportion of women $(63 \%)$ than men $(50 \%)$ reported being discouraged or intimidated in their $\operatorname{program}\left(\chi^{2}(1, N=228)=76.44, \mathrm{p}<0.001\right)$.

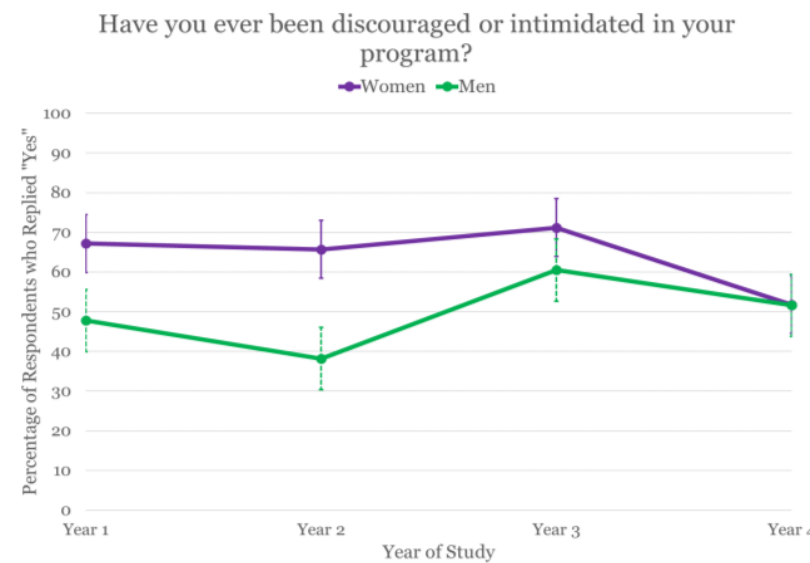

Figure 1. Discouragement and intimidation of men and women in undergraduate engineering, averaged across all institutions. (Note: Error bars indicate standard deviation.)

It is also noteworthy that about half of all respondents who identified as men had been discouraged or intimidated in their program.

Peers and instructors were consistently indicated as primary or at least major sources of discouragement, for all institutions surveyed. Figure 2 shows that an exception to this was Institution B, wherein the primary source of discouragement was indicated to be Other. Respondents did not specify who "Other" was.

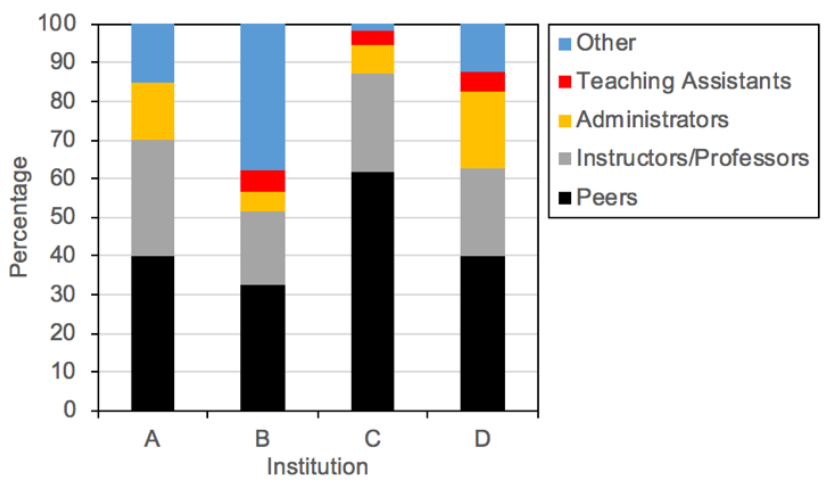

Figure 2. Sources of discouragement and intimidation per institutions. (Note: Data for Teaching Assistants was not available for Institution A.)
Respondents indicated that they engaged their instructors through e-mail or online means most of the time (averaging 37\%, ranging from 35-39\%); through talking to them before or after class (averaging 27\%, ranging from 23-39\%); and through meeting them in office hours (averaging 20\%, ranging from 18-24\%). Men and women generally interacted with their instructors in similar ways, although women were more likely than men to interact with their instructors through online means or through someone else. Men were more likely than women to interact with their instructors through talking to them before or after class and to not interact with instructors or Teaching Assistants (TAs) at all. Men and women were equally likely to meet their instructors in office hours and to not interact with their instructors but still interact with TAs.

Women self-reported having less of a chance of securing opportunities in their program more often than men did. Men reported having more of a chance of securing opportunities in their program more often than women did. Furthermore, Figure 3 shows that significantly more men than women reported having much more of a chance, and more women than men reported having much less of a chance $\left(\chi^{2}(1, N=293)=17.36, \mathrm{p}<0.05\right)$.

Relative to your peers, do you feel that you have the same chance of securing opportunities in your program?

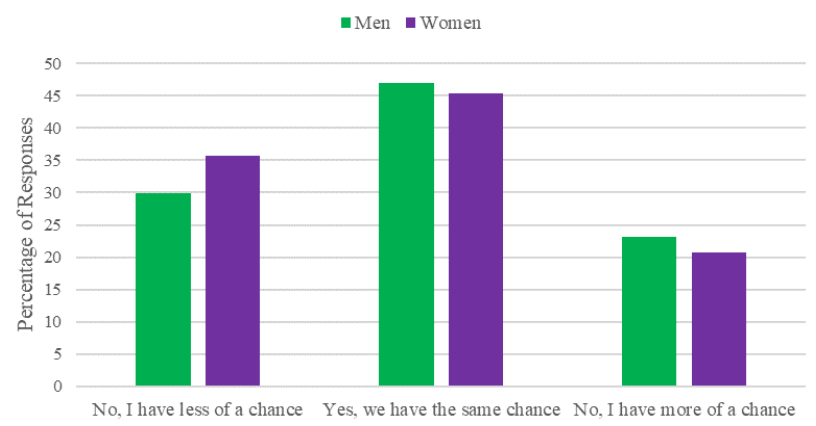

Figure 3. Men's and women's chances of securing opportunities in their programs, across all institutions.

Students were presented with a gender balanced list of comparable female and male exemplar engineers. When asked to select the names of engineering exemplars they recognized as having made contributions to the engineering field, in general, men tended to choose male exemplars and women tended to choose female exemplars, showing minor bias for their own gender. The exception to this trend was Institution $\mathrm{C}$, whose male respondents greatly preferred their own gender while female respondents showed no significant bias. At Institution C, recognition of exemplars was also related to year of study. Figure 4 shows the distribution of male and female exemplars that were identified by students, across the years of study. This shows that at Institution $\mathrm{C}$ students were more likely to know female exemplars in later years of study $\left(\chi^{2}(1, N=293)=\right.$ $39.54, \mathrm{p}=0.01)$. 


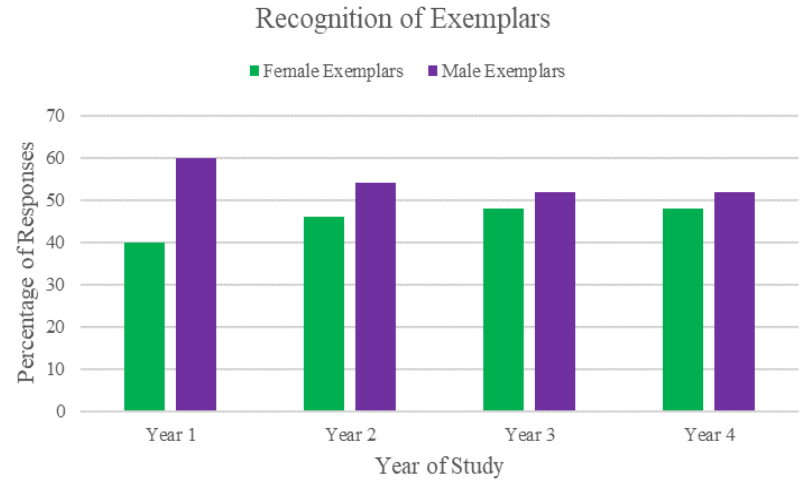

Figure 4. Recognition of exemplars across all years of study and gender, for Institution C.

Generally, women (60\%) at all institutions reported having difficulty finding group members Sometimes or Often or Always, more often than men (45\%), with the exception of Institution D. At Institution D, men reported having difficulty finding group members more often than women. For pre-designated groups, women $(78 \%)$ at Institutions $\mathrm{A}$ and $\mathrm{C}$ reported having issues working in their groups Sometimes or Often or Always, more often than men (56.5\%). In contrast, men (70.5\%) at Institutions $\mathrm{B}$ and D reported having issues working in their groups Sometimes or Often or Always, more often than women $(61.75 \%)$. The most commonly cited reasons for tension were poor communication, unfair delegation of workload, poor time management, and lack of motivation. Some women, in their comments, noted overt sexism present in pre-designated groups. Men, in their comments, did not address discrimination or gender.

Women $(47.5 \%)$ were slightly more likely than men ( $40.25 \%)$ to have someone who gives them career advice, with the exception of Institution A, where men (54\%) were more likely than women (40\%) to have someone who gives them career advice.

In terms of student profile, women (36\%) were more likely than men (24\%) to have a family member who is a practising engineer. Women and men did not generally differ in their career intentions, except in the area of pursuing further education. More men (23\%) than women (17\%) indicated that they intended to pursue further education beyond undergraduate studies. Women $(8.5 \%)$ at Institutions $\mathrm{C}$ and $\mathrm{D}$ were more likely to pursue nonengineering jobs than men (3\%).

\section{DISCUSSION}

The purpose of this study was to expand on the findings of a previous study on the experiences of women in engineering [16]. Of particular interest in this study were sources of reported discouragement, intimidation, and discrimination as well as institutional differences.

\subsection{Similarities}

In terms of similarities to previous research, it is clear that women are experiencing more discouragement and intimidation in undergraduate engineering programs than men are, especially in their middle years. This trend is consistent across all institutions studied thus far and is therefore likely a facet of larger engineering culture $[5,13$, $15,17]$. This chilly climate evidently alienates a significant portion of men as well, as their reports of discouragement and intimidation average around $50 \%$ across all years of study - an exceptionally high number. The decreases in reported levels of discouragement by fourth year seen in the pilot study have also been replicated in this study. The reasons for this are unclear, but a few comments from respondents revealed that this may be due to the development of "an uncaring attitude" or "thick skin," where the student has become desensitized to negativity around them. Alternatively, students know that the end of their program is near and may be "eager to leave, seeing the end in sight" or have developed an adequate support system by this time. This attitude change may begin in third year, as evident from the increased variability in responses to the question of discouragement for women.

Women's and men's interactions with their instructors are similar, except for within the classroom. Many female respondents reported that their professors would either "put [them] on the spot," humiliating them in front of the whole class if they answered incorrectly, or routinely let others interrupt them or repeat them without validating their contributions. This may account for why women prefer to have others approach their professors for them (i.e., to avoid discomfort) and why they prefer to meet their professors at scheduled office hours (i.e., to avoid being interrupted by peers).

Perhaps related to how often women interact with their instructors and their issues with men in their classes more generally, women more often reported having lower chances of securing opportunities in their program, as compared to their peers. The differences between men and women on this item are particularly noticeable at the extremes (i.e., Much Less Of A Chance, Much More Of A Chance). It is possible that because women feel more alienated than men in their programs, they are less likely to form wide expansive networks that could be useful when applying for jobs or participating in interesting projects. Women reported instances of sexism, including harassment, in their comments, stating that many of the peers in their program did "not take [them] seriously." Women also reported that they did not want to report others for disconcerting behaviour against them, for fear that they would be found out and ostracized by their peers or others for speaking up about the issue. It is very likely that the general competitiveness and chilly climate associated with engineering is responsible for this trend. 
Like the previous study, men and women did not generally differ in terms of career intentions and goals, with the exception that this sample had institutiondependent gender differences in the proportions of students intending to pursue graduate studies and non-engineering work. This shows that women and men both aspire to do similar work. Nevertheless, many women reported being assigned administrative duties instead of technical work when in project groups with their peers, much to their frustration.

It is notable that all respondents surveyed across the four institutions had similar things to say when asked about the most frustrating and favourite parts of their programs. Participants most often cited interest in subject matter, hands-on practical experiences, and team building as their favourite aspects of their programs, regardless of gender. Next to workload and disorganized program management, chilly climate was the most commonly reported frustration of students, regardless of gender. Similar to previous research, women reported instances of sexism (both specific events and general occurrences) that they faced on a daily basis during their program in both their frustrations with the program and in additional comments sections of the survey. Men did not report on discrimination against them, except in a couple of isolated instances, where these respondents felt that the number of women around them (when at an equal 50/50) intimidated them. No women made negative or critical comments about equity initiatives at their schools, including women who reported that they personally did not experience discrimination. Several men in the study made comments about the inappropriateness of equity initiatives, of this study itself, and of women being allowed into engineering.

These results all seem to point to meta-institutional issues in engineering culture (i.e., a chilly climate) that may be responsible for women's comparatively worse experiences in their undergraduate engineering programs.

\subsection{Differences}

In terms of differences between the present and previous research, men and women both tended to choose their own gender when given exemplars to choose from in this sample, while women in the previous study showed no significant bias. The exception to this trend was Institution C, whose results replicated the previous study's. Furthermore, it was discovered that year of study may influence students' knowledge of exemplars, with first years being the most biased against women. This suggests that different institutions - particularly, different student groups - may create an atmosphere more or less sensitive to diversity issues in representation of exemplary engineers.

Women reported having more trouble finding group members than men, with the exception of Institution D, which showed the opposite trend. Previous research found no trend. With these new results, it is probable that this particular item is dependent on institutional and student culture. Institution D, in particular, is well-known in the wider community for being committed to the inclusion of women and diverse peoples in their STEM (Science, Technology, Engineering, and Math) programs. Additionally, its engineering programs are newer than those at Institutions A, B, and C. Previous institutional biases and policies that may have been unfavourable to women do not exist to as great an extent at this institution. It is possible that peer culture, representation of women, and inclusive climate at this institution affect students' interactions with each other. The fact that men at this institution found it more difficult to find group members, compared to women, may be attributable to a mismatch between a meta-institutional chilly climate and the institution's inclusive climate. Going into engineering, men may have made assumptions based on societal stereotypes regarding engineers and, when those stereotypes were challenged by their school and their peers, they had trouble navigating the social elements of their program. Alternatively, because Institution D's programs are relatively new, students have trouble working with others simply because there are fewer students to choose from. Cohort-specific effects may contribute to this trend.

In this study, women were more likely than men to have someone who gives them career advice, with the exception of Institution A, where the trend was reversed. This is in opposition to previous research, which found no trend. These findings may point to institutional or cohort differences. It is unknown who the sources of advice are for the respondents. If they are institutionally affiliated, there may be institutional-level differences in how women are engaged by their peers, instructors, and program staff. If they are extra-institutional, such as family members, these results may be specific to the sample.

In terms of women who reported the most discrimination, Institution $\mathrm{C}$ had the greatest proportion. This result is interesting because this institution has many programs and initiatives for supporting women and minorities. It is possible that these initiatives are not well advertised (Institution D advertises its programs and initiatives consistently very well, for example, and Institution $\mathrm{C}$ may not have this kind of coverage). Alternatively, Institution C's initiatives may not be welltargeted or impactful enough for students. In any case, every institution that was part of this study had issues regarding the inclusion of women in their programs, despite every one of these institutions having programs combating stigma and a chilly culture. This suggests that these programs may not be effective. They may be underfunded, disorganized, not well targeted, not well advertised, and/or not strong enough to combat the metainstitutional chilly climate of engineering. 


\subsection{New Revelations}

This study uncovered that peers are the largest source of discouragement, intimidation, and discrimination against undergraduate engineering students, for all genders. Next to peers, instructors and professors were significant sources of negative experiences, followed by administrators, others, and TAs. It is clear that the issue of including women in engineering is not unique to an "old generation," but is something that extends to the current generation of aspiring engineers. This is further underscored by many of the young (male) students' comments about their exclusionist views on women in engineering.

The exception to the pattern of source of discouragement was Institution B, the American public university. This may be a facet of that specific institution, but it also implies a possible difference between Canadian and American issues of the inclusion of women in undergraduate engineering. This difference warrants separate study of the Canadian context, as well as further investigation into the Other category that was indicated as the primary source of discouragement in the American context.

This study also uncovered many small-scale differences between Canadian institutions, showing that at least some issues regarding the inclusion of women in engineering can be addressed at the level of the institution. There has been limited literature on the topic of institutional differences accounting for differential gender-related experiences in engineering, but what exists [4] is supported by this study.

\subsection{Limitations}

Women's backgrounds were different from men's in this sample (e.g., they were more likely to have a family member who is a practising engineer). There were fewer background differences present in previous research than in current research and it is not clear whether this presents a sample bias or whether this highlights institution- or cohort-level differences.

Genders other than male and female were not explicitly represented in this study. Although respondents could indicate whether they fall into a gender identity group apart from male or female, this group was treated as one single Other category. There were not enough respondents in this category to qualify for significant statistical analysis. Additionally, exemplars given in this survey did not include persons in this category.

Other intersectional identities were not significantly targeted and explored, but comments about race, ethnicity, and age came up in the results. These likely did not significantly impact the results, but it is possible that the sample studied was biased in such a way that one group contained more people with marginalized identities than the other.
There were not enough participants from each respective stream of engineering to make conclusion about program-specific or stream-specific experiences.

Experiences related to work terms will be covered in future work and were not covered in this study, despite programs such as co-op being highly integrated into the general engineering program experience. Participants were instructed to refer only to their experiences in the academic context and to exclude experiences with co-op, but comments about co-op and work terms still occurred.

Recruitment and data collection times for this study were very short - over a one-month period in total. This resulted in few participants. Consequently, the experiences reported in this study may be biased. However, it is notable that many similarities between samples and to previous research were found.

With future work, many of these limitations may be overcome.

\section{CONCLUSIONS}

This study found that women experience more negativity in their undergraduate engineering programs than their male peers. Furthermore, these male peers appear to be the primary source of women's discouragement, discomfort, discrimination, and intimidation.

Furthermore, this study found that institutions differ in how inclusive they make their climate to women. It is clear that, despite all institutions under study having clear guidelines and initiatives for equity and diversity, certain institutions are doing better on certain aspects of inclusion than the others.

It is clear that better equity programs and initiatives are needed to target all sources of discouragement.

\subsection{Recommendations}

Preliminary recommendations for all institutions include:

- $\quad$ survey or engage students of all genders for feedback about their undergraduate experiences, so that priorities for improvement can be identified;

- continuously monitor the successes and failures of existing and new initiatives, so that resources are used effectively;

- $\quad$ target peers and instructors for education such as bias eradication training, and make this education mandatory and repeated (i.e., crucial to cultivating institutional culture).

If every institution does their part in creating a community culture wherein equity is critical foundation, significant improvements in all students' experiences in engineering education will be made. As well, the retention 
of women in engineering beyond the undergraduate degree may improve.

\section{Acknowledgements}

We would like to thank Jennifer Ellingham for her help with this project.

\section{References}

[1] S.J. Aiston, "Whose Academy? Gender and higher education," in Researching Higher Education: International Perspectives on Theory, Policy and Practice, J.M. Case and J. Huisman, Eds. New York, NY: Routledge, 2015, pp. 80-96.

[2] Golnaz Arastoopour, Naomi C. Chesler, and David W. Shaffer, "Epistemic Persistence: A Simulation-based Approach to Increasing Participation of Women in Engineering," Journal of Women and Minorities in Science and Engineering, vol. 20, no. 3, pp. 211-234, 2014.

[3] I.D.J. Bross, "Critical levels, statistical language and scientific inference," in Foundations of statistical inference, V.P. Godambe and D.A. Sprott, Eds. Toronto, ON: Rinehart \& Winston of Canada Ltd., 1971, pp. 500-513.

[4] Elizabeth G. Creamer, When gender comes into play: Factors that distinguish colleges of engineering with above and below average enrollment of women in undergraduate engineering, American Society for Engineering Education, 2009.

[5] Emmeline de Pillis and Lisette de Pillis, "Are engineering schools masculine and authoritarian? The mission statements say yes," Journal of Diversity in Higher Education, vol. 1, no. 1, pp. 33-44, 2008.

[6] Tara C. Dennehy and Nilanjana Dasgupta, "Female peer mentors early in college increase women's positive academic experiences and retention in engineering," in Proceedings of the National Academy of Sciences, Sapna Cheryan (Ed.) (6 June 2017), vol. 114, no. 23, pp. 5964-5969.

[7] Benjamin J. Drury, John O. Siy, and Sapna Cheryan, "When Do Female Role Models Benefit Women? The Importance of Differentiating Recruitment from Retention in STEM," Psychological Inquiry, vol. 22, pp. 265-69, 2011.

[8] Xiang-Yun Du, "Gendered practices of constructing an engineering identity in a problem-based learning environment," European Journal of Engineering Education, vol. 31, no. 1, pp. 35-42, 2011.

[9] Engineers Canada, 2017 Engineering Enrolment and Degrees Awarded Report. 2018. Available from https://engineerscanada.ca/publications/canadian-engineers-fortomorrow-2017\#female-undergraduate-enrolment.

[10] Engineers Canada, Percentage of newly licensed engineers who are women [Graph], 2018. Available from https://engineerscanada.ca/reports/national-membershipreport/2018-report.

[11] Wendy Faulkner, "Nuts and Bolts and People: GenderTroubled Engineering Identities," Social Studies of Science, vol. 37, no. 3, 2007.

[12] Wendy Faulkner, "Doing gender in engineering workplace cultures. I. Observations from the field," Engineering Studies, vol. 1, no. 1, pp. 3-18, 2009.

[13] Irene F. Goodman et al., A Comprehensive Evaluation of Women in Engineering Programs. Cambridge, MA: Goodman Research Group Inc., 2002, 264 pp.

[14] Catherine Hill, Christianne Corbett, and Andresse St Rose, Why so few? Women in science, technology, engineering, and mathematics, Washington, DC: American Association of University Women, 2010.

[15] Rose M. Marra, Kelly A. Rodgers, Demei Shen, and Barbara Bogue, "Women Engineering Students and Self-Efficacy: A Multi-Year, Multi-Institution Study of Women Engineering Student Self-Efficacy," Journal of Engineering Education, vol. 98, no. 1, pp. 27-38, 2009.

[16] Natalie Mazur, Bronwyn Chorlton, and John Gales, "The Experiences of Women in Undergraduate Engineering," Proceedings from the 2018 Canadian Engineering Education Association (CEEA-ACEG18) Conference, 2018.

[17] Robert D. Reason and Susan R. Rankin, "College Students' Experiences and Perceptions of Harassment on Campus: An Exploration of Gender Differences," College Student Affairs Journal, vol. 26, no. 1, pp. 7-29, 2006.

\section{Appendix A: Sample Survey Questions}

Please answer the following questions keeping in mind your academic experiences in the classroom and at your school. If you are on a work term (co-op or

internship), please focus on your experiences at school and not at the workplace. We hope to look at work experiences in a separate study.

1. Which engineering program are you in?

2. Which year are you in, or will be entering when you return from your work term?

3 . Are you taking a voluntary 5 th year in addition to your core degree program? (Note: This does not include students who take co-op and are in their 5th year of studies but have 4 th year standing.)

- Yes

- No 
4. What is your gender?

- Male

- Female

- Other / Prefer not to say

\section{How old are you?}

6. Do you have a family member who is a practising engineer?

- Yes

- No

7. Do you have someone who provides you with career advice?

- Yes

- No

8. Of the following, choose the people that you recognize as having made contributions to the field of engineering. [Multi-select]

- List of ten randomized names, 5 female and 5 male, recommended by engineering professor contacts

9. How do you typically interact with your professors?

[Multi-select]

- E-mail and/or online

- Meeting them in office hours

- Talking to them before/after class

- Through someone else (e.g., asking a friend to talk to the professor)

- I don't interact with my professors, but talk to

Teaching Assistants (TAs) instead

- I don't interact with my professors or my TAs

10. Have you ever been discouraged or intimidated in your program?

- Yes

- No

11. Please rank how much each of the following sources contributed to your discouragement or intimidation (where 1 is the source that most significantly caused your discouragement or intimidation): [Displayed if Yes to 10]

- Peers (other students)

- Teaching Assistants (TAs)

- Professors

- Administrators

- Other

12. Relative to your peers, do you feel that you have the same chance of securing opportunities in your program?

- No, I have much less of a chance

- No, I have a little less of a chance

- Yes, we have the same chance

- No, I have a little more of a chance

- No, I have much more of a chance
13. Have you ever had difficulty finding group members when assigned group work?

- Never

- Rarely

- Sometimes

- Often

- Always

14. Have you ever encountered difficulties when working in a pre-designated group whose members you did not get to choose?

- Never

- Rarely

- Sometimes

- Often

- Always

15. Can you tell us about what kinds of difficulties you encountered? [Optional]

16. What are your plans after graduation?

- Working in an engineering field

- Working in another field (non-engineering)

- Pursuing further studies in an engineering field

- Pursuing further studies in another field (nonengineering)

- Taking time off

- Other

17. (Optional) Additional comment [Optional]

18. What is the most frustrating part of your program?

19. What is your favourite part of your program?

20. If you have any further comments about your educational experience that you wish to share, enter them below. Otherwise, click the Next button to finish the survey. [Optional] 